\title{
When Are Comparative Dynamics Monotone?
}

\author{
Mark Huggett*
}

February 14, 2002

\begin{abstract}
A common problem in dynamic economic theory is to determine when an increase in a parameter and/or an initial condition increases the future dynamics of a theoretical economy. This paper provides conditions that are necessary and sufficient for making statements of this type. The result is applicable to situations with a single agent or with many agents in the presence or absence of uncertainty. The result holds for general notions of what it means for a parameter, an initial condition or even the dynamics of a model to be increasing.
\end{abstract}

JEL Classification: C60, D90

Keywords: Comparative Dynamics, Recursive Models, Monotonicity

${ }^{*}$ I thank Karl Mosler for helpful comments. Affiliation: Georgetown University Address: Economics Department, Georgetown University, Washington DC 20057- 1036

E-mail: mh5@georgetown.edu

Homepage: http://www.georgetown.edu/faculty/mh5

Phone: (202) 687- 6683

Fax: (202) 687- 6102

Running Headline: Comparative Dynamics 


\section{Introduction}

A common problem in dynamic economic theory is to determine when an increase in a parameter and/or an initial condition increases the future dynamics of a theoretical economy. Consider three examples. In capital theory when does an increase in the discount factor or the initial capital stock increase the capital stock in all future periods? In consumer theory when does an increase in earnings risk or initial wealth lead a consumer to increase expected wealth holding in all future periods over the life cycle? In human capital theory when does an increase in the dispersion of the initial human capital distribution lead to an increase in the dispersion of the human capital distribution in all future periods over the life cycle? Such questions are the focus of a theory of monotone comparative dynamics.

This paper offers a general approach to monotone comparative dynamics questions in models with a recursive structure. The approach has two key features. First, at a point in time a consumer, firm or economy is described by a state variable. The analysis then focuses on the distribution (i.e. probability measure) of the state variable. The distribution can be interpreted in a number of ways depending on the application. For example, in single agent problems in the presence or absence of uncertainty it describes the probability that the agent (e.g. a consumer, firm or economy) will be in a particular state or collection of states in a future period as viewed from the current period. In multiple agent problems without uncertainty it describes the actual realized distribution of agents over states in a future period. In problems with a continuum of agents and idiosyncratic uncertainty the distribution also describes the actual realized distribution of agents over states. Second, these distributions have a recursive structure. To specify this let $\lambda$ denote a distribution. The recursive structure is then given by $\lambda_{j+1}=T_{j \theta} \lambda_{j}$, where the function $T_{j \theta}$ maps the period $j$ distribution into the period $j+1$ distribution and $\theta$ is a parameter governing dynamics.

The monotone comparative dynamics proposition that is the focus of this paper is given below. The proposition considers two sequences of distributions $\left\{\lambda_{j}\right\}$ and $\left\{\lambda_{j}^{\prime}\right\}$ defined recursively by $\lambda_{j+1}=T_{j \theta} \lambda_{j}$ and $\lambda_{j+1}^{\prime}=T_{j \theta^{\prime}} \lambda_{j}^{\prime}$. These sequences may differ because an initial condition differs (i.e. $\lambda_{1} \neq \lambda_{1}^{\prime}$ ) and/or because a parameter $\theta$ differs (i.e. $\theta \neq \theta^{\prime}$ ) that governs the dynamics of the economy. The proposition below states that $\lambda_{j}$ is larger than $\lambda_{j}^{\prime}$ in every period provided that this is true in the first period and that $\theta$ is larger than $\theta^{\prime}$. 


$$
\theta \geq_{\Theta} \theta^{\prime} \text { and } \lambda_{1} \succeq \lambda_{1}^{\prime} \Longrightarrow \lambda_{j} \succeq \lambda_{j}^{\prime}, j=1,2, \ldots
$$

This paper provides necessary and sufficient conditions for this proposition to hold. These conditions can be stated at various levels of abstraction each with its own merits. When these conditions are stated directly in terms of properties of the map $T_{j \theta}$ they turn out to be quite simple and to have great generality. At this level of abstraction these conditions apply to arbitrary binary relations $\succeq$ on distributions that are reflexive and transitive and to arbitrary reflexive binary relations $\geq_{\Theta}$ on parameters. This result could be interpreted as saying that at a high enough level of abstraction all monotone comparative dynamics problems are alike and have a simple structure.

To apply these results to concrete problems, it is important to develop detailed characterizations of these conditions at lower levels of abstraction for specific choices of the binary relation $\succeq$ used to order distributions. Thus, for a specific problem the process is one of reverse engineering. The map $T_{j \theta}$ is written in terms of deeper structure derived from the problem at hand. Then either sufficient or necessary conditions, stated in terms of this deeper structure, are backed out from the necessary and sufficient conditions stated in terms of the map $T_{j \theta}$.

One useful level of abstraction to consider is where the dynamics of the model can be stated in terms of properties of a decision rule and properties of an exogenous Markov shock process. A decision rule maps the state variable in a given period into a value of the decision variables. The distribution of the state then follows a Markov process determined by the decision rule and the exogenous shocks. The decision rule can be viewed as a rule of thumb, can be estimated from data or can be derived as the solution to a maximization problem. The last interpretation is familiar from the theory of dynamic programming.

When stochastic dominance is used to order distributions, it turns out that sufficient conditions for monotone comparative dynamics are easy to state in terms of properties of the decision rule and shock process. ${ }^{1}$ When the decision rule is increasing in both the state variable and the parameter governing dynamics and when the exogenous shock process is increasing in the sense of stochastic dominance both in the current shock and in the parameter then the monotone comparative dynamics proposition holds. When a decision rule is derived as a

\footnotetext{
${ }^{1}$ Stochastic dominance, sometimes called first order stochastic dominance, is a strong notion of monotone comparative dynamics. It is strong since a distribution $\lambda$ stochastically dominates a distribution $\lambda^{\prime}$ implies that the expectation of any variable that is an increasing function of the state variable is larger under $\lambda$ than under $\lambda^{\prime}$. Thus, stochastic dominance has many implications.
} 
solution to a maximization problem, this result is complementary to the work in the monotone comparative statics literature. This literature characterizes when optimal decisions are monotone in parameters that describe the objective and constraints.

It is important to emphasize at this point that comparative dynamics and comparative statics focus on different objects. Specifically, the monotone comparative statics literature characterizes when optimal decisions are monotone in parameters whereas the monotone comparative dynamics characterizes when future distributions are increasing in the initial distribution and/or a parameter affecting the dynamics. The case when distributions are ordered by stochastic dominance brings these two literatures together as comparative statics results are key to establishing assumptions on primitives so that comparative dynamics are monotone. However, for some monotone comparative dynamics problems properties beyond the monotonicity of decision rules and shock processes are essential. This will be the case when some binary relation other than stochastic dominance is used to order distributions. The last section of the paper provides an example illustrating this point.

The remainder of the paper is organized as follows. The literature related to this paper is briefly outlined in section 1.1. The framework for monotone comparative dynamics is described in section 2. The results are stated and proved in section 3. The results are applied in several examples in section 4 .

\section{$1.1 \quad$ Related Literature}

This paper is related to work in three areas. First, Hopenhayn and Prescott (1992) analyze when stationary distributions (i.e. steady states) exist, are unique and increase in a parameter. Their work and the work in this paper are related as both focus on distributions as the unit of analysis and both make use of monotonicity properties of functions mapping a set of distributions into itself. Both lines of research are complementary in that one characterizes steady states, whereas the other characterizes comparative dynamics. The monotone comparative dynamics proposition provided here is applicable regardless of whether or not steady states exist. An important application where steady states are not relevant is the class of problems focusing on consumer behavior over the life cycle. Second, this paper is complementary to the work in the monotone comparative statics literature (see Milgrom and Shannon (1996) or Topkis (1998)). This literature offers techniques to characterize when optimal decisions are increasing in 
parameters describing objectives and constraints. As remarked previously, the monotone comparative statics literature links up perfectly with the monotone comparative dynamics results of this paper when stochastic dominance is used to order distributions. Third, there is a literature on comparative dynamics within capital theory. The standard problem in this literature (see Becker and Boyd (1997)) is to compare how the capital stock varies over time in deterministic models when a parameter (i.e. tax rate or a discount factor) is increased or an initial condition (i.e. the capital stock) is increased. The comparative dynamics framework developed here covers problems of this type as a special case.

\section{$2 \quad$ Framework}

The framework is based on the following assumptions: (i) a function $T_{j \theta}$ maps the set $\Lambda(X, \mathcal{X})$ into itself, where $\Lambda(X, \mathcal{X})$ is the set of probability measures defined on a measurable space $(X, \mathcal{X})$ and $X$ is called the state space and (ii) there is a reflexive and transitive binary relation $\succeq$ on $\Lambda(X, \mathcal{X})$ and a reflexive binary relation $\geq_{\Theta}$ on the parameter space $\Theta$. It is sometimes useful to state the mapping $T_{j \theta}$ in terms of other objects. For example, equation (1) states this mapping in terms of a transition function $P_{j \theta}$. Transition functions are a standard tool in the theory of Markov processes. The transition function on the state variable is defined in equation (2) in terms of a decision rule $y(x, j ; \theta)$ and a transition function $\pi_{j \theta}$ for a Markov shock process. In this context, the state variable is $x=(y, z)$ and the state space is specialized to be $X=Y \times Z$, where $Y$ is the set of possible values of the decision variable and $Z$ is the set of possible values of a shock. This specialized formulation of the state space is widely used in the dynamic models presented in Stokey and Lucas (1989).

$$
\begin{gathered}
\lambda_{j+1}(B)=T_{j \theta} \lambda_{j}(B) \equiv \int_{X} P_{j \theta}(x, B) d \lambda_{j}, \forall B \in \mathcal{X} \\
\lambda_{j+1}(B)=T_{j \theta} \lambda_{j}(B) \equiv \int_{X} \pi_{j \theta}\left(z,\left\{z^{\prime}:\left(y(x, j ; \theta), z^{\prime}\right) \in B\right\}\right) d \lambda_{j}, \forall B \in \mathcal{X}
\end{gathered}
$$

Definition 1 defines terms. Stochastic dominance and transition functions are given the following interpretations. Stochastic dominance describes when one distribution is larger than another in the sense that more probability or 
weight is put on high realizations. ${ }^{2}$ A transition function $P(x, B)$ states the probability that next period's state will lie in $B$ given that this period's state is $x$. The definition of comparative dynamics is that described in the introduction. A different but related notion of economic dynamics occurs when the sequence defined by $\lambda_{j+1}=T_{j \theta} \lambda_{j}$ is monotone in time. For this notion, a useful proposition would be $\lambda_{j+1} \succeq \lambda_{j} \Longrightarrow \lambda_{j+2} \succeq \lambda_{j+1}$. When $T_{j \theta}$ is time invariant, necessary and sufficient conditions for this proposition follow from the results of this paper.

DEFINITION 1: Let $\left(X, \geq_{X}\right)$ be a partially ordered set, $(X, \mathcal{X})$ be a measurable space and $\Lambda(X, \mathcal{X})$ be the set of probability measures on $(X, \mathcal{X})$.

(i) A set $B \subseteq X$ is increasing provided $y \in B$ whenever $y \geq_{X} x, y \in X$ and $x \in B$.

(ii) For each $\lambda, \lambda^{\prime} \in \Lambda(X, \mathcal{X}), \lambda$ stochastically dominates $\lambda^{\prime}$ (denoted $\lambda \succeq_{s d} \lambda^{\prime}$ ) provided $\lambda(B) \geq \lambda^{\prime}(B)$ for all increasing sets $B \in \mathcal{X}$.

(iii) $P: X \times \mathcal{X} \rightarrow[0,1]$ is a transition function provided (i) $\forall x \in X, P(x,$.$) is a$ probability measure on $(X, \mathcal{X})$ and (ii) $P(., B)$ is a $\mathcal{X}$ measurable function $\forall B \in \mathcal{X}$.

(iv) Comparative dynamics are monotone provided for all $\theta, \theta^{\prime} \in \Theta$, for all $\lambda_{j}, \lambda_{j}^{\prime} \in \Lambda(X, \mathcal{X})$ and for all $j$ the following proposition holds: $\theta \geq_{\Theta} \theta^{\prime}$ and $\lambda_{j} \succeq \lambda_{j}^{\prime} \Longrightarrow \lambda_{j+k} \succeq \lambda_{j+k}^{\prime}, k=0,1, \ldots$

\section{Results}

All the results in the paper build upon Theorem 1 . Theorem 1 states that $T_{j \theta}$ satisfying assumptions A1-2 is necessary and sufficient for the monotone comparative dynamics proposition stated in the introduction and defined in Definition 1 .

$$
\begin{aligned}
& \left.A 1 \text { ( } T_{j \theta} \text { increases in } \theta\right) \forall \theta, \theta^{\prime} \in \Theta, \theta \geq_{\Theta} \theta^{\prime} \Longrightarrow T_{j \theta} \lambda \succeq T_{j \theta^{\prime}} \lambda, \forall \lambda \in \Lambda(X, \mathcal{X}) \text {. } \\
& \text { A2 ( } T_{j \theta} \text { preserves order) } \forall \lambda, \lambda^{\prime} \in \Lambda(X, \mathcal{X}), \lambda \succeq \lambda^{\prime} \Longrightarrow T_{j \theta} \lambda \succeq T_{j \theta} \lambda^{\prime}, \forall \theta \in \Theta
\end{aligned}
$$

${ }^{2}$ An equivalent definition of stochastic dominance (see Lehmann 1955 or Shaked and Shanthikumar 1994) is as follows: $\lambda$ stochastically dominates $\lambda^{\prime}$ provided $\int f(x) d \lambda \geq \int f(x) d \lambda^{\prime}$ for all measurable, increasing functions $f$ for which the integrals exist. Note that the definition of stochastic dominance is based on a partially ordered set $\left(X, \geq_{X}\right)$. A partial order $\geq_{X}$ is reflexive, transitive and antisymmetric. 
THEOREM 1: Assume $\succeq$ is a reflexive and transitive binary relation on $\Lambda(X, \mathcal{X}), \geq_{\Theta}$ is a reflexive binary relation on $\Theta$ and $T_{j \theta}: \Lambda(X, \mathcal{X}) \rightarrow \Lambda(X, \mathcal{X})$ for all $(j, \theta) \in\{1,2, \ldots\} \times \Theta$.

Comparative dynamics are monotone if and only if $T_{j \theta}$ satisfies A1-2.

Proof:

$(\Longrightarrow)$ A1 follows from the definition of monotone comparative dynamics by letting $\lambda_{j}=\lambda_{j}^{\prime}$, whereas A2 follows by letting $\theta=\theta^{\prime}$.

$(\Longleftarrow)$ It is sufficient to show that $\theta \geq_{\Theta} \theta^{\prime}$ and $\lambda_{j} \succeq \lambda_{j}^{\prime} \Longrightarrow T_{j \theta} \lambda_{j} \succeq T_{j \theta^{\prime}} \lambda_{j}^{\prime}$. This is established in the equation below. The leftmost inequality below holds by A2. The rightmost inequality below holds by A1. $T_{j \theta} \lambda_{j} \succeq T_{j \theta^{\prime}} \lambda_{j}^{\prime}$ then holds by transitivity.

$$
\lambda_{j+1}=T_{j \theta} \lambda_{j} \succeq T_{j \theta} \lambda_{j}^{\prime} \succeq T_{j \theta^{\prime}} \lambda_{j}^{\prime}=\lambda_{j+1}^{\prime}
$$

Theorem 2 restates Theorem 1 in terms of properties of transition functions. The result is that a transition function that is increasing in the state $x$ and in the parameter $\theta$ is a necessary and sufficient condition for comparative dynamics to be monotone when distributions are ordered by stochastic dominance. The proof is based on showing that $P_{j \theta}$ increasing in $\theta$ is equivalent to $T_{j \theta}$ increasing in $\theta$ and that $P_{j \theta}$ increasing in $x$ is equivalent to $T_{j \theta}$ preserving order. Thus, key properties of the map $T_{j \theta}$ are easily restated in terms of properties of the transition function $P_{j \theta}$.

$B 1$ ( $P_{j \theta}$ increases in $\left.\theta\right) \forall \theta, \theta^{\prime} \in \Theta, \theta \geq_{\Theta} \theta^{\prime} \Longrightarrow P_{j \theta}(x,.) \succeq_{s d} P_{j \theta^{\prime}}(x,),. \forall x \in X$.

B2 $\left(P_{j \theta}\right.$ increases in $\left.x\right) \forall x, x^{\prime} \in X, x \geq_{X} x^{\prime} \Longrightarrow P_{j \theta}(x,.) \succeq_{s d} P_{j \theta}\left(x^{\prime},.\right), \forall \theta \in \Theta$

THEOREM 2: Assume $\left(X, \geq_{X}\right)$ is a partially ordered set, $\geq_{\Theta}$ is a reflexive binary relation on $\Theta, P_{j \theta}$ is a transition function for all $(j, \theta) \in\{1,2, \ldots\} \times \Theta$, $T_{j \theta}$ is defined by equation (1) and stochastic dominance is the binary relation on distributions.

Comparative dynamics are monotone if and only if $P_{j \theta}$ satisfies B1-2.

Proof: The result follows from Theorem 1 and Lemma 1.

LEMMA 1: Assume $\left(X, \geq_{X}\right)$ is a partially ordered set, $\geq_{\Theta}$ is a reflexive binary relation on $\Theta, P_{j \theta}$ is a transition function for all $(j, \theta) \in\{1,2, \ldots\} \times \Theta$, $T_{j \theta}$ is defined by equation (1) and stochastic dominance is the binary relation on distributions. 
(i) $P_{j \theta}$ satisfies $B 1$ if and only if $T_{j \theta}$ satisfies $A 1$.

(ii) $P_{j \theta}$ satisfies $B 2$ if and only if $T_{j \theta}$ satisfies A2.

Proof:

(i) Show B1 if and only if A1. The definition of $T_{j \theta}$ in equation (1) and assumption B1 imply that $T_{j \theta} \lambda(B)=\int P_{j \theta}(x, B) d \lambda \geq \int P_{j \theta^{\prime}}(x, B) d \lambda=T_{j \theta^{\prime}} \lambda(B)$ for all increasing sets $B \in \mathcal{X}$ because for all values of $x$ and $B$ the integrand $P_{j \theta}(x, B) \geq P_{j \theta^{\prime}}(x, B)$. To show the reverse implication, let $\lambda$ be the measure putting all mass on a single point $x$.

(ii) Show B2 if and only if A2. A2 is restated as $\lambda \succeq_{s d} \lambda^{\prime} \Rightarrow \int P_{j \theta}(x, B) d \lambda \geq$ $\int P_{j \theta}(x, B) d \lambda^{\prime}$ for all increasing sets $B \in \mathcal{X}$. Two facts establish that B2 implies A2. First, $\lambda \succeq_{s d} \lambda^{\prime}$ implies $\int f(x) d \lambda \geq \int f(x) d \lambda^{\prime}$ for all measurable and increasing functions $f$ for which the integrals exist. See Lehmann (1955) or Shaked and Shanthikumar (1994, Ch. 1) for a proof. Second, $P_{j \theta}(x, B)$ is an increasing function of $x$ for all increasing sets $B \in \mathcal{X}$ and the integral of this function clearly exists. To show the reverse implication, let $\lambda$ and $\lambda^{\prime}$ be measures putting all mass on a single point $x$ and $x^{\prime}$ respectfully, where $x \geq_{X} x^{\prime}$.

Theorem 3 is useful in many applications. In Theorem 3 the mapping $T_{j \theta}$ and the transition function $P_{j \theta}$ are defined directly in terms of a decision rule and an exogenous Markov shock process (see equation (2) in section 2). Many of the dynamic models considered in Stokey and Lucas (1989) can be posed in terms of decision rules and exogenous shock processes. Theorem 3 states that if the decision rule $y(x, j ; \theta)$ and transition probabilities $\pi_{j \theta}$ are increasing in the parameter $\theta$ (assumption $\mathrm{C} 1$ ) and if the decision rule and transition probabilities are increasing in $x$ and $z$ (assumption $\mathrm{C} 2$ ) then comparative dynamics are monotone. The proof is based on observing that the two properties in $\mathrm{C} 1$ are reinforcing in producing a transition function $P_{j \theta}$ that increases in the parameter $\theta$ (assumption B1) and that the two properties in $\mathrm{C} 2$ are reinforcing in making $P_{j \theta}$ increase in the state $x$ (assumption B2). ${ }^{3}$

In Theorem 3 it is important to note that (i) the state space has a product representation $X=Y \times Z$, where $Y$ and $Z$ can be interpreted as the endogenous and exogenous components of the state variable, (ii) a decision rule $y: X \times$

\footnotetext{
${ }^{3}$ Conditions $\mathrm{C} 1$ and $\mathrm{C} 2$ are sufficient but not necessary for producing monotone comparative dynamics. To see this point consider an example. Let $Y=Z=\Theta=\{0,1\}$ with the usual orders. Let $y(x, j ; \theta)$ and $\pi_{j \theta}$ be arbitrary as long as $y(x, j ; \theta) \in Y, \forall x, j, \theta . P_{j \theta}$ is then trivially monotone in $x$ and $\theta$ when the measurable sets $\mathcal{X}, \mathcal{Y}, \mathcal{Z}$ consist of only the empty set and the entire set.
} 
$\{1,2, \ldots\} \times \Theta \rightarrow Y$ is considered which is increasing in that $x \geq_{X} x^{\prime}$ implies $y(x, j ; \theta) \geq_{Y} y\left(x^{\prime}, j ; \theta\right)$, (iii) $\pi_{j \theta}: Z \times \mathcal{Z} \rightarrow[0,1]$ is a transition function defined on the exogenous shocks, (iv) $\pi_{j \theta}$ increasing in $z$ or $\theta$ always means in the sense of stochastic dominance and (v) $\left(X, \geq_{X}\right)$ has the component order (i.e. $x \geq_{X} x^{\prime}$ provided $\left(y \geq_{Y} y^{\prime}\right.$ and $\left.z \geq_{Z} z^{\prime}\right)$, where $\left.x=(y, z), x^{\prime}=\left(y^{\prime}, z^{\prime}\right)\right)$.

C1: $y(x, j ; \theta)$ increases in $\theta, \forall(x, j)$ and $\pi_{j \theta}$ increases in $\theta, \forall(j, z)$.

C2: $y(x, j ; \theta)$ increases in $x$ and $\pi_{j \theta}$ increases in $z, \forall(j, \theta)$.

C3: $y(x, j ; \theta)$ is measurable in $x, \forall(j, \theta)$.

C4: $\left(X, \geq_{X}\right),\left(Y, \geq_{Y}\right),\left(Z, \geq_{Z}\right)$ are partially ordered sets, $(X, \mathcal{X}),(Y, \mathcal{Y})$, $(Z, \mathcal{Z})$ are measurable spaces, $\geq_{\Theta}$ is a reflexive binary relation on $\Theta, X=$ $Y \times Z, \geq_{X}$ is the component order and $\mathcal{X}$ is the product $\sigma$-algebra.

THEOREM 3: Assume that $T_{j \theta}$ is defined by equation (2), stochastic dominance is the binary relation on distributions and the regularity conditions C3-4 hold.

Then $y(x, j ; \theta)$ and $\pi_{j \theta}$ satisfy C1-2 imply that comparative dynamics are monotone.

Proof: First, note that since $y(x, j ; \theta)$ is measurable (assumption C3) then Stokey and Lucas (1989, Theorem 9.13) implies that $T_{j \theta}$ defined in equation (2) maps $\Lambda(X, \mathcal{X})$ into itself. Second, let the transition function $P_{j \theta}$ be constructed as indicated in equation (2). Then $\mathrm{C} 1$ gives sufficient conditions for $P_{j \theta}$ to satisfy $\mathrm{B} 1$ and $\mathrm{C} 2$ gives sufficient conditions for $P_{j \theta}$ to satisfy B2. Thus, Theorem 3 follows from Theorem 2 .

\section{Examples}

The results from the previous section are now illustrated by several examples. Examples 1 and 2 are single agent problems with uncertainty which are drawn from the literatures on optimal growth and commodity storage. Theorem 3 of this paper is applied to both examples. ${ }^{4}$ Example 3 highlights the point that

\footnotetext{
${ }^{4}$ The reader will observe that shocks are independent in both example 1 and 2. Clearly, this is not important for applying Theorem 3. However, it is useful since existing results from these literatures, which have been established for the case of independent shocks, can immediately be used to make comparative dynamic statements.
} 
often interesting monotone comparative dynamics problems require that some binary relation other than stochastic dominance orders distributions and the point that when this is the case properties of decision rules beyond monotonicity are needed. In this example the conclusions are established by a direct application of the general result proved in Theorem 1. Example 4 shows how to map a heterogeneous-agent model into the comparative dynamics framework.

Example 1: Optimal Growth - Brock and Mirman (1972)

The optimal growth problem is to maximize $E\left[\sum_{j=0}^{\infty} \beta^{j} u\left(z_{j} f\left(k_{j}\right)-k_{j+1}\right)\right]$ subject to the constraint $0 \leq k_{j+1} \leq z_{j} f\left(k_{j}\right)$. In this problem $z_{j} f\left(k_{j}\right)$ is the production function in period $j$, which depends on capital $k_{j}$ and an independent and identically distributed shock $z_{j}$ with distribution $\pi$. The state $x=(y, z)$ consists of the capital stock $y=k$ and the shock $z$. Brock and Mirman (1972, Lemma 1.1-1.2) establish that the optimal decision rule $y(x, j ; \theta)$ for capital brought into the next period is a continuous and time-invariant function of the state and increases in each component of the state. Danthine and Donaldson (1981, Theorem 5.1 ) establish that $y(x, j ; \theta)$ is an increasing function of the discount factor $\theta=\beta$. Given these properties of the decision rule and shocks, Theorem 3 implies that the expected path of capital or output over time is always weakly greater with higher values of the discount factor $\beta$ and/or higher values of the initial capital stock. These results complement the comparative steady state analysis provided for this model by Danthine and Donaldson (1981).

Example 2: Commodity Storage - Deaton and Laroque (1992)

Deaton and Laroque (1992) study the problem of competitive storage of a commodity where harvests are independent and identically distributed and the market demand curve is downward sloping in the quantity consumed. The state $x=(y, z)$ consists of beginning-of-period inventory $y$ and harvest $z$. They prove that there is a unique rational expectations equilibrium with profit maximizing storage. Furthermore, they prove that the inventory decision $y(x, j ; \theta)$ is (i) a continuous, increasing and time-invariant function of the state $x$ and (ii) an increasing function of the discount factor $\theta$ at which future payoffs are discounted. Based on these results, Theorem 3 implies that expected future inventories are increasing in the discount factor starting from any distribution of the state and that expected future inventories are increasing in the initial inventory and harvest.

Example 3: Precautionary Wealth Accumulation - Huggett (2001)

One of the fundamental questions in the theory of precautionary savings is when do increases in earnings risk lead to increases in an agent's expected wealth 
accumulation at each age over the life cycle? The underlying decision problem that agent's face is the standard problem in the literature that formalizes the permanent-income hypothesis (e.g. Schechtman (1976)). For this problem the state is $x=(y, z)$, where $y$ is wealth and $z$ is an earnings shock, and the parameter $\theta$ indexes earnings processes $\pi_{j \theta}$ that are independent over periods. ${ }^{5}$ The amount of wealth that is carried from one period to the next is described by a decision rule $y(x, j ; \theta)$.

Huggett (2001) answers this question both at the level of properties of decision rules and properties of preferences by using the general approach to monotone comparative dynamics advanced in this paper. He uses the increasingconvex stochastic order rather than stochastic dominance. ${ }^{6}$ The increasingconvex stochastic order is better suited for determining when expected wealth accumulation increases with increases in earnings risk. Heuristically, this is because the distribution of wealth may be more dispersed as earnings risk increases at the same time that mean wealth holding increases. The increasing-convex stochastic order allows two such distributions to be ordered whereas stochastic dominance does not.

Huggett (2001) uses the general result in Theorem 1 of this paper to show that when the decision rule for wealth accumulation $y(x, j ; \theta)$ is increasing and convex in the state $x$ and increasing in earnings risk $\theta$ then comparative dynamics are monotone. An implication is that expected wealth accumulation at each age over the life cycle increases with increases in risk and/or with increases in initial wealth holding.

A sketch of the argument behind this result is to note first that $T_{j \theta}$ increases in $\theta$ (assumption A1) is equivalent to the three expressions below, where the functions $f$ are those that are increasing and convex. The expression $E_{j \theta}[f \mid x] \equiv$ $\int f\left(x^{\prime}\right) P_{j \theta}\left(x, d x^{\prime}\right)$ is simply a conditional expectation. Huggett notes that the rightmost inequalities hold when $E_{j \theta}[f \mid x]$ increases in $\theta$ and this in turn holds when $y(x, j ; \theta)$ increases in $\theta$ and $\pi_{j \theta}$ increases in $\theta$ in the sense of increasing risk described earlier.

\footnotetext{
${ }^{5}$ The partial order $\geq_{\Theta}$ on earnings processes is a natural generalization of the definition of increasing risk provided in Rothschild and Stiglitz (1970). In particular, one earnings process is riskier than another provided that period by period one distribution is riskier than another in the Rothschild-Stiglitz sense.

${ }^{6} \lambda$ dominates $\lambda^{\prime}$ in the increasing-convex order provided $\int f d \lambda \geq \int f d \lambda^{\prime}$ for all measurable $f$ that are increasing and convex and for which the integrals exist. Since $f(x)=y$ is an increasing and convex function representing wealth holding, $\lambda$ dominates $\lambda^{\prime}$ in the increasing-convex order implies that expected wealth holding is higher under $\lambda$ than under $\lambda^{\prime}$.
} 


$$
T_{j \theta} \lambda \succeq T_{j \theta^{\prime}} \lambda \Leftrightarrow \int f d T_{j \theta} \lambda \geq \int f d T_{j \theta^{\prime}} \lambda \Leftrightarrow \int E_{j \theta}[f \mid x] d \lambda \geq \int E_{j \theta^{\prime}}[f \mid x] d \lambda
$$

The remainder of the argument is to note that $T_{j \theta}$ preserves order (assumption A2) is again equivalent to the three expressions below. Huggett then notes that the rightmost inequalities hold when $E_{j \theta}[f \mid x]$ is increasing and convex in $x$ and that this holds when $y(x, j ; \theta)$ is increasing and convex in $x$.

$$
T_{j \theta} \lambda \succeq T_{j \theta} \lambda^{\prime} \Leftrightarrow \int f d T_{j \theta} \lambda \geq \int f d T_{j \theta} \lambda^{\prime} \Leftrightarrow \int E_{j \theta}[f \mid x] d \lambda \geq \int E_{j \theta}[f \mid x] d \lambda^{\prime}
$$

Example 4: Growth with Idiosyncratic Shocks - Huggett (1997)

Huggett (1997) generalizes the standard, one-sector, competitive growth model to include incomplete markets and idiosyncratic labor endowment risk. There is a continuum of agents of mass equal to one who are homogeneous in preferences but possibly heterogeneous in period labor endowment and the holdings of a risk-free asset. An agent's state variable is $x=(y, z)$, where $y$ is current asset holding and $z$ is a Markovian labor endowment shock with transition probability $\pi_{\theta}$. The distribution of agents over states is denoted $\lambda$, whereas $\theta$ denotes a parameter entering agent's preferences and/or Markovian endowment process. An equilibrium in this model is $\left(c(x, \lambda ; \theta), y(x, \lambda ; \theta), W(\lambda), R(\lambda), T_{\theta}(\lambda)\right)$ such that (i) $c, y$ are optimal decision rules, given $W, R$ and $T$, (ii) $W, R$ are competitive wages and gross interest rates, (iii) $c, y$ are feasible in that consumption and next period's asset holding summed over the population equal available total output $F(\lambda)$ (i.e. $\left.\int_{X}(c(x, \lambda ; \theta)+k(x, \lambda ; \theta)) d \lambda=F(\lambda), \forall \lambda\right)$ and (iv) $T_{\theta}$ is a law of motion, defined below, mapping this period's distribution into next period's distribution.

$$
\begin{aligned}
& \lambda^{\prime}(B)=T_{\theta} \lambda(B) \equiv \int_{X} P_{\lambda \theta}(x, B) d \lambda, \forall B \in \mathcal{X} \\
& P_{\lambda \theta}(x, B) \equiv \pi_{\theta}\left(z,\left\{z^{\prime}:\left(y(x, \lambda ; \theta), z^{\prime}\right) \in B\right\}\right)
\end{aligned}
$$

Clearly, Theorem 1 provides necessary and sufficient conditions for monotone comparative dynamics in this model. However, since the distribution $\lambda$ enters the transition function $P_{\lambda \theta}$ above but not in the framework described previously, Theorems 2 and 3 are not applicable. With this said, it turns out that it is straightforward to verify that (i) $P_{\lambda \theta}$ increases in $\theta$ is sufficient for $T_{\theta}$ to increase 
in $\theta$ (assumption A1) and that (ii) $P_{\lambda \theta}$ increases in $(x, \lambda)$ (using the coordinate order) is sufficient for $T_{\theta}$ to preserve order (assumption A2). Thus, a version of Theorem 2 can be established that offers sufficient conditions for monotone comparative dynamics for this example. Using this result it is then easy to state sufficient conditions in terms of properties of decision rules and exogenous Markov processes along the lines of Theorem 3. In particular, $y(x, \lambda ; \theta)$ increasing in $(x, \lambda)$ and $\pi_{\theta}$ increasing in $z$ in the sense of stochastic dominance is sufficient for assumption A2 to hold, while $y(x, \lambda ; \theta)$ increasing in $\theta$ and $\pi_{\theta}$ increasing in $\theta$ is sufficient for assumption A1 to hold. 


\section{REFERENCES}

Becker, R. and J. Boyd (1997), Capital Theory, Equilibrium Analysis and Recursive Utility, (Blackwell Publishers, Oxford).

Brock, W. and L. Mirman (1972), Optimal Economic Growth and Uncertainty: The Discounted Case, Journal of Economic Theory, 4, 497- 513.

Danthine, J. and J. Donaldson (1981), Stochastic Properties of Fast vs. Slow Growing Economies, Econometrica, 49, 1007- 1033.

Deaton, A. and G. Laroque (1992), On the Behavior of Commodity Prices, Review of Economic Studies, 59, 1-23.

Hopenhayn, H. and E. Prescott (1992), Stochastic Monotonicity and Stationary Distributions for Dynamic Economies, Econometrica, 60, 1387-1406.

Huggett, M. (1997), The One-Sector Growth Model with Idiosyncratic Shocks: Steady States and Dynamics, Journal of Monetary Economics, 39, 385- 403.

Huggett, M. (2001), Precautionary Wealth Accumulation, Georgetown University, http://www.georgetown.edu/faculty/mh5/.

Lehmann, E. (1955), Ordered Families of Distributions, Annals of Mathematical Statistics, 26, 399-419.

Milgrom, P. and C. Shannon (1994), Monotone Comparative Statics, Econometrica, 62, 157- 80.

Rothschild, M. and J. Stiglitz (1970), Increasing Risk I: A Definition, Journal of Economic Theory, 2, 225- 43.

Schechtman, J. (1976), An Income Fluctuation Problem, Journal of Economic Theory, 12, 218- 41.

Shaked, M. and J. Shanthikumar (1994), Stochastic Orders and Their Applications, (Academic Press, New York).

Stokey, N. and R. Lucas with E. Prescott (1989), Recursive Methods in Economic Dynamics, (Harvard University Press, Cambridge).

Topkis, D. (1998), Supermodularity and Complementarity, (Princeton University Press, Princeton). 